\title{
EL FORO DEL DOMICILIO DEL EMPLEADOR EN LOS CONTRATOS INTERNACIONALES DE TRABAJO. UN APUNTE SOBRE LA SENTENCIA DE LA SALA DE LO SOCIAL DEL TSJ DE MADRID DE 17 DE ABRIL DE 2020
}

\author{
THE FORUM OF THE EMPLOYER'S DOMICILE \\ IN INTERNATIONAL LABOUR CONTRACTS. A NOTE \\ ON THE JUDGMENT OF THE SOCIAL CHAMBER OF THE \\ HIGH COURT OF JUSTICE OF MADRID OF 17 APRIL 2020
}

\author{
Unai BeLintXon Martin \\ Profesor Contratado Doctor de Derecho Internacional Privado \\ Universidad Pública de Navarra (UPNA)
}

Recibido: 12.11.2020 / Aceptado: 04.12.2020

DOI: https://doi.org/10.20318/cdt.2021.5984

\begin{abstract}
Resumen: Interposición de Recurso de Suplicación frente a Sentencia del Juzgado de lo Social que desestima en instancia la demanda interpuesta por despido al apreciar de oficio la incompetencia territorial de los Juzgados y Tribunales españoles para conocer del hecho controvertido. Falta de competencia apreciada inadecuadamente, y con clara confusión en la delimitación de las relaciones forum-ius, por el Juzgado de instancia tras el análisis del derecho sustantivo cubano.

Palabras clave: Competencia judicial internacional, Derecho europeo, domicilio del empleador, Reglamento Bruselas I bis, contratos individuales de trabajo.

Abstract: Appeal against the judgment of the Social Court dismissing at first instance the action for dismissal on the ground that the Spanish courts did not have jurisdiction to hear the case of their own motion. Lack of jurisdiction, assessed inadequately, and with clear confusion in the delimitation of forum-ius relations, by the Court of First Instance after analysis of cuban substantive law.

Keywords: Jurisdiction, European Law, Employer's domicile, recast Brussels I Regulation, individual contracts of employment.

Sumario: I. Consideración preliminar. II. Hechos. III. Cuestiones controvertidas y un apunte sobre Derecho internacional privado. 1. El foro del domicilio del empleador y RBI bis 2 . Un breve apunte sobre el foro del domicilio del empleador y la LOPJ. IV. Consideración final.
\end{abstract}

\section{Consideración preliminar}

1. El 17 de abril de 2020, la Sección $4^{\text {a }}$ del Tribunal Superior de Justicia de Madrid (Sala de lo Social) estimó mediante Sentencia núm. 210/2020 un recurso de suplicación surgido en procedimiento de despido ante el Juzgado de lo Social número 7 de Madrid en el que de forma concreta se solicitaba la declaración de competencia judicial internacional y territorial del citado Juzgado de instancia para 
conocer de la demanda de despido, y tras ello, la devolución de las actuaciones para emitir o dictar nueva sentencia resolviendo sobre el fondo de la pretensión ejercitada por la parte demandante.

2. Indicado lo anterior, el presente comentario tiene por objeto una vez expuestos los hechos acaecidos, analizar las cuestiones más controvertidas y su posible incidencia sobre nuestra disciplina de Derecho internacional privado.

\section{Hechos}

3. A la luz de los antecedentes expuestos en la Sentencia núm. 210/2020 de la Sección $4^{\text {a }}$ del Tribunal Superior de Justicia de Madrid (Sala de lo Social), la parte actora, ciudadano cubano, afiliado a UGT, dado de alta y cotizando en la Seguridad Social Cubana, que ha prestado servicios como auxiliar administrativo en Cuba a la Agencia Española de Cooperación Internacional (AECID) adscrita al Ministerio de Asuntos Exteriores, Unión Europea y Cooperación español, y percibiendo un salario bruto mensual de 1.166,59 \$ con inclusión de la parte proporcional de las pagas extras mediante un contrato de suministro de fuerza de trabajo suscrito entre AECID y la empresa estatal cubana PALCO, interpone demanda por despido frente a ambas (si bien se advierte en la demanda interpuesta que considera la verdadera empleadora a AECID y que únicamente demanda a PALCO para prever o contrarrestar una potencial alegación de falta de litisconsorcio pasivo necesario).

4. La citada demanda fue desestimada mediante sentencia en primera instancia por el Juzgado de lo Social referido al apreciar de oficio la incompetencia territorial de los Juzgados y Tribunales españoles para conocer de la demanda interpuesta por la representación del trabajador, absolviendo a la empresa estatal cubana empleadora PALCO y a la AECID española de todos los pedimentos en su contra.

5. El recurso de suplicación interpuesto por la actora, recurrente ante la Sala de lo Social del TSJ de Madrid, fue impugnado por el Abogado del Estado en representación de la AECID. La codemandada no ha comparecido a juicio, ni ha impugnado el recurso interpuesto.

6. El Juzgador de instancia basa su decisión de desestimación en la falta de competencia judicial internacional de los Juzgados y Tribunales españoles al considerar acreditado que según el derecho cubano (Decreto-ley $n^{\circ}$ 55/1982 sobre la contratación del personal de servicios en representaciones extranjeras en la República de Cuba) la prestación de servicios a representaciones extranjeras por personas naturales residentes en Cuba únicamente pueden materializarse concertando un contrato con una empresa estatal cubana, puesto que la prestación de servicios directamente a una delegación extranjera es motivo de sanción penal según el derecho sustantivo o material cubano. En efecto, el juzgador de instancia concluye que debe interpretarse que el contrato se ha concertado con la empresa estatal cubana PALCO y no con AECID, por lo que esta última no ostentaría legitimación pasiva para ser demandada.

7. Los artículos 2, 5 y la disposición especial única de la norma cubana referida son del siguiente tenor:

Art. 2: Los ciudadanos cubanos y los extranjeros residentes permanentes en el país no podrán realizar labores, administrativas, técnicas o de servicios, incluyendo las domésticas o de cualquier otro tipo, a representaciones extranjeras, si no han sido objeto de contrato entre la citada representación extranjera y la empresa estatal correspondiente.

Art.5: El pago de los servicios contratados se efectúa por la representación extranjera sobre la base de las regulaciones establecidas, con cargo a los saldos existentes en las cuentas corrientes abierta a nombre de la representación extranjera en el Banco Nacional de Cuba.

Disposición especial única: Se considera actividad económica ilícita la prestación de servicios personales, sean éstos de naturaleza administrativa, técnica o de cualquier otra, incluido el servicio doméstico, a las representaciones extranjeras a que se refiere el Artículo 1 de este Decreto-Ley, por ciudadanos 
cubanos o por extranjeros residentes permanentes en nuestro país, cuando esta prestación se contrate directamente por dichos trabajadores con la representación extranjera.

La conducta referida en el párrafo anterior es sancionada de acuerdo con la legislación penal vigente.

\section{Cuestiones controvertidas y un apunte sobre Derecho internacional privado.}

8. De lo expuesto en la sentencia analizada de la Sala de lo Social TSJ de Madrid se refleja claramente el desacuerdo con la resolución de instancia. Muy acertadamente la Sala en el primer fundamento de derecho de su sentencia se dedica a esclarecer la confusión en la delimitación de las relaciones forum-ius ${ }^{l}$ proyectada por el Juzgado de instancia en su sentencia al advertir que [...] Resulta prioritario deshacer la confusión en la que se ha incurrido, y así es necesario distinguir la competencia judicial internacional, que implica la determinación de si deben conocer de un asunto los Jueces españoles o los extranjeros, y es por tanto una cuestión de extensión y límites de la Jurisdicción española, y de otra parte la determinación de la Ley aplicable a las obligaciones contractuales, aspecto que, evidentemente, es distinto y lógicamente posterior, no previo, al problema de decidir qué jurisdicción nacional es la competente para conocer del asunto. El Juez nacional que resulte competente habrá de resolver el litigio aplicando su derecho nacional propio, o el extranjero que proceda, según las normas de derecho internacional privado.

9. Resuelta esta cuestión, no menor, el debate jurídico principal lo centra la Sala en sus fundamentos de derecho tercero y cuarto. Es decir, en primer lugar, si ante el supuesto planteado AECID debe o no considerarse empleadora ${ }^{2}$, y en segundo lugar, estando AECID domiciliada en España si los Juzgados y Tribunales españoles tienen competencia judicial internacional para conocer de la controversia suscitada por los servicios prestados por el trabajador en la citada representación extranjera en Cuba.

10. La Sala no duda sobre la aplicación al caso del Reglamento Bruselas I bis ${ }^{3}$ y proyecta en sus fundamentos de derecho tercero y cuarto expresamente la siguiente valoración:

Fundamento de Derecho Tercero: [...] En el presente caso ya es de aplicación el Reglamento (UE) $n^{\circ} 1215 / 2012$ del Parlamento Europeo y del Consejo, de 12 de diciembre de 2012, relativo a la competencia judicial, el reconocimiento y la ejecución de resoluciones judiciales en materia civil y mercantil es norma sumamente relevante para la materia ("Bruselas I Bis"), en vigor para las acciones judiciales entabladas a partir del 10-1-2015. Este reglamento (al igual que su precedente "Bruselas I") establece como criterio de su aplicación y a la vez determinante de la competencia el del domicilio del demandado, con independencia de que el demandante no tenga la nacionalidad de un estado miembro o que el litigio no presente vínculos con otros estados miembros de la UE, como sucede en el presente caso. En lo que ahora interesa, si el demandado tiene su domicilio en un estado de la UE, es aplicable el reglamento Bruselas I bis y resulta competente la jurisdicción del estado miembro donde el demandado tiene su domicilio [...]

Fundamento de Derecho Cuarto: Sobre el artículo 21.1 y el RBI bis [...] Artículo 21.1. Los empresarios domiciliados en un Estado miembro podrán ser demandados: a) ante los órganos jurisdiccionales del Estado en el que estén domiciliados [...] No es dudoso, conforme a lo que se viene exponiendo, que la AECID, al tener su domicilio en España, estado miembro de la UE, puede ser demandada ante los órganos judiciales de nuestro país. Y ello con independencia de lo que se resuelva sobre cuál es el derecho aplicable y la solución que proceda en el plano sustantivo.

\footnotetext{
${ }^{1}$ Sobre la cuestión pueden verse el excelente estudio del profesor J.D. GonZÁlez CAMPos, "Las relaciones entre forum y ius en el Derecho Internacional Privado", Anuario español de Derecho internacional, n 4, 1977-1978, pp. 89-136. También puede verse una referencia focalizada sobre el Derecho del transporte de la cuestión en: J.J. Álvarez RuBio, "Transporte Marítimo Internacional de Mercancías: alcance material y conflictual de la Autonomía contractual”, AEDIPR., t. VIII, 2008, pp. 558-559.

${ }^{2}$ O. Fotinopoulou Basurko, El proceso laboral internacional en el derecho comunitario, Sevilla, Consejo Económico y Social de Andalucía, 2008, pp. 107 y ss.

${ }^{3}$ Respecto a la pluralidad de bloques normativos coexistentes y la cascada de la especificidad, puede verse J.J. ÁLVAREZ RuBio, "Las reglas de especificidad como cauce para superar los conflictos normativos entre el Derecho comunitario y los Convenios internacionales en materias especiales", La Ley, 29 de octubre de 2010, pp. 1-6.
} 
En cuanto a la codemandada empresa estatal cubana Empleadora PALCO, se trata de una entidad extracomunitaria que no tiene su domicilio en un estado miembro de la UE y en concreto no lo tiene en España. El actor tampoco ha prestado servicios en España y por ello no puede ser de aplicación lo previsto en el art. 21.2 en relación con el propio art. 21.1.b) recién transcrito. Con arreglo al art. 6, si el demandado no está domiciliado en un Estado miembro, la competencia judicial se regirá, en cada Estado miembro, por la legislación de ese Estado miembro, y ello nos llevaría a la aplicación del art. 25 de la LOPJ, con arreglo al cual no se cumple ninguno de los criterios para poder demandar a PALCO en España.

Sin embargo, se ha de tener presente que la demanda solamente se ha dirigido contra PALCO a efectos de prevenir una posible alegación de falta de litisconsorcio pasivo necesario, y que el suplico de la demanda solamente pide la condena de la AECID a la readmisión como consecuencia del despido nulo que dicha entidad española ha realizado, según el demandante, quien no pide pronunciamiento alguno en relación con PALCO.

11. En efecto, de la lectura combinada de los artículos 21 y 23 del RBI bis se proyectan distintos foros elegibles para aquellos supuestos en los que el trabajador sea el demandante ${ }^{4}$ : Así, en primer lugar y a tenor de lo dispuesto por los artículos 21.1.a y 20.2 del RBI bis los empleadores/empresarios domiciliados en un Estado miembro pueden ser demandados ante los tribunales del Estado miembro de su domicilio ${ }^{5}$. Entendiéndose que aquellos empresarios no domiciliados en la UE pero con sucursales, agencias o filiales abiertas en territorio de la Unión están domiciliados en el Estado Miembro de la UE en el que se encuentre dicha sede. Por otro lado, y según lo preceptuado por el artículo 21.1.b.i el trabajador podrá demandar ante el órgano jurisdiccional del lugar en el que o desde el cual el trabajador desempeñe habitualmente su trabajo ${ }^{6}$ ante el órgano jurisdiccional del último lugar en que lo haya desempeñado . $^{7}$ Otra opción sería, para aquellos casos en los que el trabajador no desempeña o no ha desempeñado habitualmente su trabajo en un único Estado, interponer la correspondiente acción ante el órgano jurisdiccional del lugar en que esté o haya estado situado el establecimiento que haya empleado al trabajador (artículo 21.1.a.ii) ${ }^{8}$. También cabría la posibilidad para los trabajadores desplazados en el sentido de la Directiva 96/71/CE 9 , modificada por la Directiva UE/2018/95710 y según su artículo 6 (norma de transposición España: Ley 45/199911 -artículo 16.1-), interponer la acción correspondiente ante los tribunales del Estado en el cual esté prestando servicios el trabajador desplazado temporalmente ${ }^{12}$. Y finalmente, según el artículo 23 del RBI bis, cabe la posibilidad de quedar sometidos mediante acuerdo atributivo de jurisdicción a unos órganos jurisdiccionales determinados y elegidos por empleado y empleador ${ }^{13}$. Eso sí, siempre y cuando el acuerdo atributivo de jurisdicción sea posterior al nacimiento del litigio o

${ }^{4}$ A.L. Calvo Caravaca / J. Carrascosa González, Derecho internacional privado, Vol. II, 2018, Granada, Comares, pp. 1144-1152.

${ }^{5}$ Sobre el foro general del domicilio del demandado, pueden verse las consideraciones realizadas acerca del contrato de embarque internacional en su obra: J.L. IrIARTE ÁngeL, El contrato de embarque internacional, Madrid, Beramar S.L., 1993, pp. 100 y ss.

${ }^{6}$ J.L. Iriarte Ángel, "La precisión del lugar habitual de trabajo como foro de competencia y punto de conexión en los Reglamentos europeos", CDT, Octubre 2018, Vol. 10, n² 2, pp. 488-495.

${ }^{7}$ J.F. Lousada Arochena / R.P. Ron Latas, El contrato de trabajo internacional, Valladolid, Lex Nova, 2013, pp. 52-53

${ }^{8}$ Respecto al ámbito del desplazamiento transfronterizo de trabajos, véase con incidencia sobe el sector de la ley aplicable el excelente estudio de la profesora y amiga M. CASADO ABARQUERo, "Legislación aplicable a los trabajadores desplazados en el marco de una prestación de servicios en la Unión Europea”, J.L. GoÑ SeIN / J.L. IRIARTe Ángel (Dirs), Prevención de riesgos laborales y protección social de trabajadores expatriados, Cizur Menor, Thomson Reuters Aranzadi, 2019, pp. 339-369

${ }^{9}$ Directiva 96/71/CE del Parlamento Europeo y del Consejo de 16 de diciembre de 1996 sobre el desplazamiento de trabajadores efectuado en el marco de una prestación de servicios. [DOUE L 81/1 de 21 de enero de 1997].

${ }^{10}$ Directiva (UE) 2018/957 del Parlamento Europeo y del Consejo de 28 de junio de 2018 que modifica la Directiva 96/71/ CE sobre el desplazamiento de trabajadores efectuado en el marco de una prestación de servicios. [DOUE L 173/16 de 9 de julio de 2018].

${ }^{11}$ Ley 45/1999, de 29 de noviembre, sobre desplazamiento de trabajadores en el marco de una prestación de servicios transnacional. [BOE $\mathrm{n}^{\circ} 286$ de 30 de noviembre de 1999].

${ }^{12}$ M.C. Vaquero LóPez, "Mecanismos de Derecho Internacional Privado Europeo para la protección de los trabajadores en supuestos de deslocalización de empresas", $A E D I P r, \mathrm{n}^{\circ}$ 17, 2017, pp. 425-471.

${ }^{13}$ U. Belintxon Martín, "Dumping Social, desarmonización socio-laboral y Derecho internacional privado: la des-Unión Europea", $A E D I P r$, t. XVI, 2016, pp. 611-642. 
permita al trabajador formular demandas ante órganos jurisdiccionales distintos de los indicados en el comentado artículo 21 del RBI bis ${ }^{14}$.

\section{1) El foro del domicilio del empleador y RBI bis}

12. Acierta de plano el TSJ de Madrid al observar la competencia de los Juzgados y Tribunales españoles para conocer del supuesto planteado en lo dispuesto por el artículo 21.1.a del RBI bis. Un foro muy utilizado en la realidad práctica por parte de personas trabajadoras nacionales españolas, e incluso extranjeras, que prestan servicios como empleadas para la Administración Pública española y sus organismos dependientes ${ }^{15}$, muchas de ellas desempeñando habitualmente su trabajo en un tercer Estado. Nuestra jurisprudencia es firme al considerar en sus resoluciones de forma reiterada que la Administración Pública española, el Ministerio de Asuntos Exteriores para los casos de embajadas y consulados y otros, tiene sin ningún género de dudas su domicilio en España.

13. En consecuencia, las personas que prestan servicios en tales representaciones españolas en el extranjero pueden articular acción judicial para la defensa de sus derechos ante los tribunales españoles. Así lo dicta el referido artículo 21.1.a del RBI bis, y así lo ha aplicado correctamente la Sala de lo Social del Tribunal Superior de Justicia de Madrid, desplazando además la aplicación del artículo 25 LOPJ, en esta sentencia comentada de 17 de abril de 2020, y en otras anteriores, como por ejemplo en su sentencia de 16 de noviembre de $2004^{16}$ en la que, aunque con cierta confusión respecto al Convenio de Bruselas de 1968, en el párrafo 12 de su Fundamento de Derecho Único de forma correcta y clarificadora indica que [...] "Encontrándonos pues, dentro del ámbito de aplicación material y espacial del Convenio, éste es plenamente aplicable, en cuanto normativa que desplaza, en situaciones como la que se analiza, las soluciones contenidas en la LOPJ, las cuales serían de aplicación para el supuesto de inexistencia de Convenios que regularan la materia", y en su sentencia 26 de septiembre de $2005^{17}$ en la que previo análisis de la nulidad del acuerdo atributivo de jurisdicción recogido en contrato laboral por incumplir los requisitos impuestos por el artículo 21 del RBI (actual artículo 23 RBI bis), aplica correctamente el artículo 19.1 del RBI (hoy artículo 21.1.a del RBI bis) otorgando competencia judicial internacional a los tribunales españoles. Sin duda es clarificador su Fundamento de Derecho cuarto al recoger que "[...] Se trata, como se ve, de foro electivo a favor de los trabajadores demandantes, por lo que los Tribunales españoles del orden jurisdiccional social sí cuentan con competencia judicial internacional para conocer de la controversia que separa a los litigantes, habida cuenta que el domicilio del Departamento ministerial demandado radica en España, y sin que a ello sea óbice el dato de que pudieran haber planteado también alguna reclamación judicial ante los Tribunales italianos, dado el lugar de su prestación de servicios y el carácter electivo del foro que ya señalamos.".

14. Así lo ha entendido también la Sala de lo Social del Tribunal Supremo, entre otras, en su sentencia de 12 de junio de $2003^{18}$ que con reiterada cita jurisprudencial al TJUE vino a declarar competentes a los Juzgados y Tribunales españoles, aunque los trabajadores fueran de nacionalidad colombiana, prestaren servicios en Colombia y fuesen residentes en Colombia puesto que el demandado era un Ministerio español. Concluye la Sala del Tribunal Supremo muy acertadamente en su Fundamento de Derecho Tercero, párrafos III, IV y V que:

\footnotetext{
${ }^{14}$ U. Belintxon Martín, "Capítulo XIV. Contratos internacionales de trabajo y competencia judicial internacional: La perspectiva del Tribunal Supremo", en A.L. Calvo Caravaca / J. Carrascosa GonzÁlez (coords.), El Tribunal Supremo y el derecho internacional privado, Vol. 1, Tomo 1, 2019, pp. 321-334

${ }^{15}$ Sobre la delimitación del concepto: M. Virgós Soriano / F.J. Garcimartín AlfÉrez, Derecho Procesal Civil Internacional. Litigación Internacional, Cizur Menor, Thomson Civitas, 2007, p. 184.

${ }^{16}$ STSJ de Madrid (Sala de lo Social), 16 noviembre 2004, [CENDOJ. ECLI: ES: TSJM: 2004:14142].

${ }^{17}$ STSJ de Madrid (Sala de lo Social), 26 septiembre 2005, [CENDOJ. ECLI: ES: TSJM: 2005:953].

${ }^{18}$ STS (Sala de lo Social) 12 junio 2003. [Aranzadi. RJ 2003\4585].
} 
[...] El Convenio de Bruselas establece un fuero general, único y excluyente: el del domicilio del demandado sito en el territorio de la Comunidad Europea, al que se circunscribe su ámbito espacial. A tal efecto, su artículo 2 dispone que: «salvo lo dispuesto en el presente Convenio, las personas domiciliadas en un Estado contratante estarán sometidas, sea cual fuere su nacionalidad, a los órganos jurisdiccionales de dicho Estado». Dicha previsión se corresponde con la preocupación manifestada por los países signatarios en su preámbulo de «fortalecer en la Comunidad la protección jurídica de las personas establecidas en la misma» (así lo recuerda el TJCE en sentencia de 13-7-1993 [ TJCE 1993, 119], núm. C-125/1992) y con la creencia de que es en el Estado de su domicilio donde mejor podrá defenderse el demandado. El fuero del domicilio del demandado es pues vinculante, salvo en los supuestos excepcionales previstos en los artículos 5.1 (competencias especiales, con fueros electivos para el demandante) y 16 (competencias exclusivas con diversos fueros obligatorios) que no son de interés para el debate [...]

[...] Además, el citado fuero general se impone necesariamente en su ámbito, cualesquiera que sean los elementos de extranjería que confluyan en la controversia [...] Consiguientemente el Convenio de Bruselas resulta aplicable al presente caso, aunque los demandantes sean de nacionalidad colombiana y residan en Colombia.

[...] De lo expuesto se sigue que el art. 25.1 LOPJ cede ante el Convenio Bruselas. Y que los fueros alternativos que dicho precepto establece (lugar de la prestación de servicios, lugar de celebración del contrato y nacionalidad española de ambas partes contratantes) sólo son válidos fuera del ámbito material y espacial de dicho Convenio.

15. Más recientemente la Sala del Tribunal Supremo se vuelve a pronunciar en similares términos en su sentencia de 16 de enero de $2018^{19}$ manteniendo el criterio anterior e indicando expresamente en su Fundamento de Derecho Tercero con independencia del desplazamiento de los trabajadores a Argelia que:

[...] Téngase presente también que las normas del Reglamento cuya infracción se denuncia lo que pretenden, precisamente es proteger a la parte contratante más débil mediante reglas de competencia más favorables a los intereses de esa parte, STJUE 10 septiembre de 2015. En nuestro caso, habida cuenta del lugar en que han sido contratados, de su nacionalidad, del domicilio social de la primera empresa contratante y del carácter instrumental de la empleadora formal (la que, al cabo, despide) es claro que el Reglamento permite que la demanda se presente ante los Tribunales españoles. Es el foro general (domicilio del empleador) el que activan los trabajadores y el que debe considerarse competente.

\section{2) Un breve apunte sobre el foro del domicilio del empleador y la LOPJ}

16. En relación a esta cuestión, merece un mero apunte con la única pretensión de desplazar la aplicación del artículo 25 de la LOPJ invocado en el pleito de la sentencia comentada y que resuelve muy acertadamente la Sala del TSJ de Madrid. Debe recordarse que dicho artículo 25 LOPJ se activará únicamente en aquellos casos en los que no sean aplicables ni las normas convencionales de las que forma parte España, ni las normas contenidas en el RBI bis que para este caso sí son de aplicación ${ }^{20}$.

17. En efecto la activación de la LOPJ responde exclusivamente a aquellos supuestos que no estén cubiertos por el RBI bis o por cualquier otra norma internacional ${ }^{21}$.

18. Recuérdese además que tal y como hemos expuesto las embajadas españolas, consulados y otros, dependientes del Ministerio de Asuntos Exteriores español, pueden ser demandadas mediante la activación del foro del domicilio demandado ante los tribunales españoles siendo el artículo 21.1.a del

\footnotetext{
${ }^{19}$ STS (Sala de lo Social), 16 enero 2018. [Aranzadi. ECLI: ES: TS: 2018:375].

${ }^{20}$ Sobre esta cuestión, entre otras: STS, Sala de los Social, de 4 de junio de 1986. [Aranzadi. RJ/1986/3460]. Y, STS, Sala de lo Social, de 14 de julio de 1988. [Aranzadi RJ/1988/5825].

${ }^{21}$ J.C. FernÁndez RozAs, “El 60 aniversario de los Tratados de Roma: algo más que una simple celebración”, La Ley, 46, 2017, pp. 1-8.
} 
RBI bis el instrumento normativo europeo plenamente invocable y aplicable por parte de los Tribuna$\operatorname{les}^{22}$. No cabe aplicar en estos casos el artículo 25 de la LOPJ, pues como advertimos anteriormente los instrumentos internacionales aplicables en España y los Reglamentos Europeos desplazan la aplicación de las normas de producción interna ${ }^{23}$. En efecto, si el demandado tiene su domicilio para este tipo de controversias en territorio español, la competencia judicial de los tribunales españoles debe sostenerse sobre el artículo 21.1.a del RBI bis y no sobre el artículo 25 LOPJ como proyecta la jurisprudencia analizada anteriormente.

\section{Consideración final}

19. La Sala de lo Social del TSJ de Madrid revierte adecuadamente el pronunciamiento de instancia al estimar el recurso interpuesto en súplica por el trabajador y declarando la competencia judicial internacional y territorial del Juzgado de $\operatorname{los}$ Social $\mathrm{n}^{\circ} 7$ de Madrid para conocer de la demanda y resolver sobre el fondo del asunto. La Sala acierta de plano al imponer al juzgador de instancia el resolver el pleito suscitado teniendo en consideración la activación del foro del domicilio del empleador del artículo 21.1.a según los criterios hermenéuticos establecidos por la doctrina jurisprudencial estatal y del TJUE, puesto que AECID dependiente del Ministerio de Asuntos Exteriores español tiene su domicilio en España.

\footnotetext{
${ }^{22}$ J.L. IRIARTE ÁNGEL, "El contrato de trabajo internacional: algunas cuestiones relacionadas con la competencia judicial internacional", Cursos de Derecho Internacional y Relaciones Internacionales, Universidad del País Vasco, Madrid, Tecnos, 2001, pp. 119 y ss.

${ }^{23}$ Sobre el artículo 25 LOPJ y su aplicación, entre otras: STS, Sala de lo Social, de 25 de octubre de 1989. [Aranzadi. $\mathrm{RJ} / 1989 / 7433]$
} 